\title{
DETERMINAÇÃO DO FATOR PARA CÁLCULO DO VALOR DAS PRESTAÇÕES PERIÓDICAS DE UM FINANCIAMENTO
}

\author{
José Zanella \\ Departamento de Matemática. Centro de Ciências Naturais e Exatas. \\ UFSM - Santa Maria, RS. \\ Giovani Gilberto Socal. \\ Fundação Regional de Economia. Santa Maria, RS.
}

\section{RESUMO}

Este artigo aborda a determinação analítica de um fator "F", utilizado para cálculo do valor das prestações nas vendas a prazo. Esse fator, que é calculado em função das condições de venda, fornece o valor das prestações periódicas ao ser multiplicado pelo preço do bem vendido.

Inicialmente faz-se a dedução da fórmula geral do fator" $F "$ e, posteriormente, apresenta-se alguns casos particulares.

Por fim simula-se exemplos hipotéticos para facilitar o entendimento.

\section{SUMARY}

ZANELLA, J., Determination of the Factor for the Value of the Periodic Installments Calculation of the Loan

This paper approaches the analytic determination of a factor "F" used for calculating the value of the installments on credit sales. The factor which is calculated according to the sale conditions, provides the value of the periodic installments as being multiplied by the price of the sold goods or served service.

At first we subtract the general formula from the " $\mathrm{F}$ " factor and, afterwards we show some particular cases.

Finally, we simulate hypothetical examples to make understanding easer. 


\section{1- DETERMINACÃO DO FATOR "F"}

Seja o seguinte fluxo de caixa genérico representativo de uma operação de venda a prazo, sob o ponto de vista do vendedor:

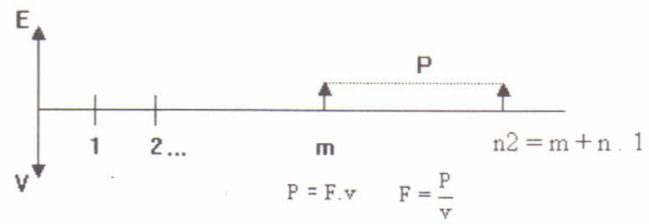

onde:

V é o valor do bem;

E é o valor da entrada, que pode ou não ser um percentual de $\mathrm{V}$;

P é o valor da prestação periódica;

n1 é o número de periodos de capitalização transcorridos entre a venda e o recebimento da primeira prestação (prazo de carência):

n é o número de prestações periódicas;

n2 é o período de pagamento da última prestação;

i é a taxa unitária efetiva de juros no periodo das prestações

Fazendo a equivalência dos valores no periodo 0 :

$$
\begin{aligned}
& V=E+\frac{P}{(1+i)^{n+1}}+\frac{P}{(1+i)^{n+1}}+\frac{P}{(1+i)^{m+2}}+\ldots+\frac{P}{(1+i)^{n+n+1}} \\
& V=E+P\left[\frac{1}{(1+i)^{n !}}+\frac{1}{(1+i)^{n+1}}+\frac{1}{(1+i)^{m+2}}+\ldots+\frac{1}{(1+i)^{n+n-1}}\right]
\end{aligned}
$$

O termo entre colchetes é a soma dos termos de uma progressão geométrica cujo primeiro termo é $a_{1}=\frac{1}{(1+i)^{n 1}}$, o último é $a_{n}=\frac{1}{(1+i)^{n+n-1}}$ e a razão éq $=\frac{1}{(1+i)} \cdot($ Veras, 1993)

A soma destes termos é chamada de fator de valor atual FVA( $\mathrm{i}, \mathrm{n}$ ), assim:

$$
F V A(i, n)=\frac{\frac{1}{(1+i)^{n 1}}-\frac{1}{(1+i)} \cdot \frac{1}{(1+i)^{n 1+n-1}}}{1-\frac{1}{(1+i)}}=\frac{\frac{1}{(1+i)^{n 1}}-\frac{1}{(1+i)^{n 1+n}}}{\frac{1+i-1}{(1+i)}}=\frac{(1+i)^{n}-1}{(1+i)^{n 1+n}} \cdot \frac{(1+i)}{i}=\frac{(1+i)^{n}-1}{i(1+i)^{n 1+n-1}}
$$


Então:

$V=E+P \cdot \frac{(1+i)^{n}-1}{i(1+i)^{n 1+n-1}}$

$P=(V-E) \cdot \frac{i(1+i)^{n 1+n-1}}{(1+i)^{n}-1}$

$F=\frac{P}{V} \quad \Rightarrow \quad F=\frac{V-E}{V} \cdot \frac{i(1+i)^{n 1+n-1}}{(1+i)^{n}-1}$

\section{1.- CASOS PARTICULARES}

1) Venda sem entrada $(E=0)$

$$
F=\frac{i(1+i)^{n 1+n-1}}{(1+i)^{n}-1}
$$

2) Venda sem entrada e sem carência $\left(E=0, n_{1}=1\right)$ :

$$
F=\frac{i(1+i)^{n}}{(1+i)^{n}-1}
$$

3) Venda com uma entrada igual a um percentual do valor da compra ( $E=r . V)$ :

$$
F=(1-r) \cdot \frac{i(1+i)^{n 1+n-1}}{(1+i)^{n}-1}
$$

4) Venda com entrada igual a um percentual $\mathbf{r}$ do valor do bem e sem carência $\left(E=r . v, n_{1}=1\right)$ :

$$
F=(1-r) \cdot \frac{i(1+i)^{n}}{(1+i)^{n}-1}
$$

5) Venda com entrada igual à prestação $(E=P)$

$\mathrm{Na}$ equação ( I ) $\operatorname{com} \mathrm{E}=\mathrm{P}$ 


$$
\begin{aligned}
& V=P+P \cdot \frac{i(1+i)^{n}-1}{(1+i)^{n 1+n-1}}=P\left[1+\frac{i(1+i)^{n}-1}{(1+i)^{n 1+n-1}}\right] \\
& F=\frac{P}{V} \quad \Rightarrow \quad F=\frac{1}{1+\frac{i(1+i)^{n}-1}{(1+i)^{n 1+n-1}}} \quad \text { ou } \quad F=\frac{(1+i)^{n 1+n-1}}{(1+i)^{n}\left[(1+i)^{n-1}+i\right]-1}
\end{aligned}
$$

6) Venda com entrada igual à prestação e sem carência $\left(E=P, n_{1}=1\right)$

$$
F=\frac{1}{1+\frac{(1+i)^{n}}{i(1+i)^{n}-1}} \quad \text { ou } \quad F=\frac{(1+i)^{n+1}-1}{i(1+i)^{n}}
$$

\section{2.- EXEMPLOS DE APLICAÇÃO}

A seguir são apresentados alguns exemplos da utilização do fator para cálculo da prestação.

2.1) Preço da mercadoria $=V=R \$ 45.000,00$

$$
\begin{aligned}
& \text { Entrada }=\mathrm{E}=\mathrm{R} \$ 12 \cdot 000,00 \\
& \text { Carência }=\mathrm{n}_{1}=3 \text { meses } \\
& \mathrm{n}=4 \text { prestações }
\end{aligned}
$$

Taxa de juros $=\mathrm{i}=22 \%$ a.m.

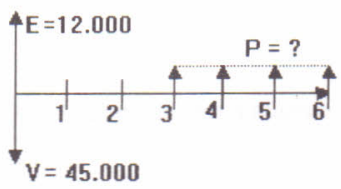

Determinar o valor do fator $\mathrm{F}$ e da

prestação

$$
\text { Expressão ( II ) } \quad \begin{aligned}
\mathrm{F} & =\frac{\mathrm{V}-\mathrm{E}}{\mathrm{V}} \cdot \frac{\mathrm{i}(1+\mathrm{i})^{\mathrm{n} 1+\mathrm{n}-1}}{(1+\mathrm{i})^{\mathrm{n}}-1}=\frac{45.000-12.000}{45.000} \cdot \frac{0,22(1+0,22)^{3+4-1}}{(1+0,22)^{4}-1} \\
\mathrm{~F} & =0,437711 \Rightarrow \quad \mathrm{P}=\mathrm{F} \cdot \mathrm{V}=0.437711 .45 .000 \quad \Rightarrow \\
\mathbf{P} & =\mathbf{R} \$ \mathbf{1 9 . 6 9 7 , 0 0}
\end{aligned}
$$

2.2) $V=R \$ 210,00$

$$
F=\frac{210-38}{210} \cdot \frac{0,25(1+0,25)^{1+4-1}}{(1+0,25)^{4}-1}[\text { expressão I ] }
$$

$\mathrm{E}=\mathrm{R} \$ 38,00$

$\mathrm{n}_{1}=1$ mês ( sem carência )

$$
\begin{aligned}
& F=0,346819 \\
& P=0,346819.210 \\
& P=R \$ 72,83
\end{aligned}
$$$$
\mathrm{i}=25 \% \text { a.m. }
$$ 
2.3) $\mathrm{V}=$ qualquer valor

$$
F=(1-0,3) \cdot \frac{0,08(1+0,08)^{2+6-1}}{(1+0,08)^{4}-1}[\text { expressão V] }
$$

$\mathrm{E}=30 \%$ de $\mathrm{V}$

$\mathrm{n}_{1}=2$ meses

$F=0.2662$

$\mathrm{n}=6$ prestações

$P=0.2662 . \mathrm{V}$

$\mathrm{i}=8 \% \mathrm{a} \cdot \mathrm{m}$

2.4) $\mathrm{V}=$ qualquer valor

$$
F=\frac{0,082(1+0,082)^{5}}{(1+0,082)^{5}-1} \quad[\text { expressão IV] }
$$

$\mathrm{E}=0$ ( sem entrada $)$

$\mathrm{n} 1=1$ quadrimestre

$$
F=0,251778
$$

$\mathrm{n}=5$ prestações quadrimestrais

$\mathrm{i}=8,2 \%$ ao. quadrimestre

$$
\mathrm{P}=0,251778 . \mathrm{V}
$$

ou seja, a prestação será da ordem de $25,1778 \%$ do valor à vista

2.5) Venda em 7 prestações iguais e sucessivas, sendo a primeira de entrada

$$
\begin{array}{ll}
\mathrm{V}=\text { qualquer valor } & \mathrm{F}=\frac{1}{1+\frac{(1+0,197)^{6}-1}{0,197 \cdot(1+0,197)^{6}}} \text { [expressão VIII] } \\
\mathrm{E}=\mathrm{P} & \\
\mathrm{n} \mathrm{I}=1 \text { mês } & \mathrm{F}=0,229862 \\
\mathrm{n}=6 \text { prestações } & \\
\mathrm{i}=19,7 \% \text { a.m } & \mathrm{P}=0,229862 . \mathrm{V}
\end{array}
$$

o que significa que a prestação será da ordem de $22,9862 \%$ do valor da mercadoria.

\section{3.- AUTOMAÇÃO DOS CALCULOS}

O cálculo do fator " $F$ " pode ser automatizado através da utilização de fluxogramas simples, adiante apresentados. Note-se que o valor do fator não depende fundamentalmente dos valores absolutos de V, E e P, mas sim das relações entre estes valores dessa forma, no segundo caso, o cálculo de $\mathrm{F}$ se baseia na relação entre $\mathrm{E}$ e $\mathrm{V}$, podendo ser utilizado para todas as operações que se enquadrem na relação dada. Do mesmo modo, no terceiro caso o cálculo considera $E=P$ e o fator assim obtido pode servir para todas as vendas que obedecem esta condição. 
Cabe, portanto, observar que os fluxogramas do $2^{\circ}$ e $3^{\circ}$ caso pode ser adaptados - e inclusive fundidos num mesmo algoritmo, se desejado - de tal sorte que permitem, através da combinação dos dados $\mathbf{r}, \mathbf{n} 1, \mathbf{n}$ e i, a elaboração de tabelas de fatores " $F$ " que possam ser utilizados nas diversas condições de venda à prazo da empresa.

3.1) $\underline{1}^{\circ} \mathrm{CASO}: \quad$ Não se conhece a relação entre $\mathrm{Ve} \mathrm{E}$ (valores quaisquer).

Argumentos de entrada $\mathrm{V}, \mathrm{E}, \mathrm{n}_{1}, \mathrm{n}, \mathrm{i}$

Resultados $\mathrm{P}$

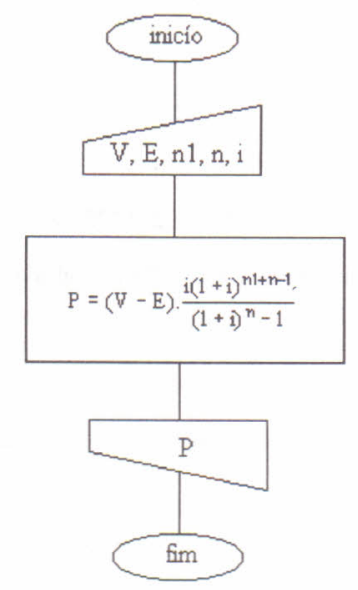

3.2. $\underline{2}^{\circ}$ CASO A entrada é uma parcela conhecida do valor total $(E=r . V)$

Argumentos de entrada $r, n_{1}, n, i$

Resultados F

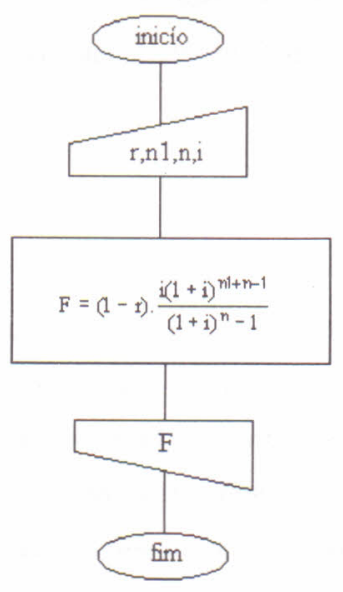


3.3. $-\underline{3^{\circ}} \underline{\mathrm{CASO}} \quad \mathrm{A}$ entrada é igual à prestação $(E=P)$

Argumentos de entrada $\mathrm{n} 1, \mathrm{n}, \mathrm{i}$

Resultados F

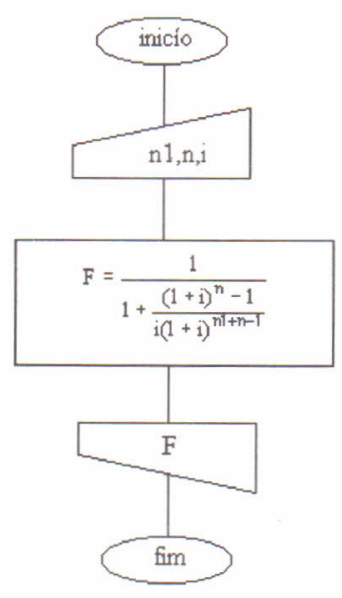

\section{CONCLUSÃO:}

O resultado deste trabalho é uma fórmula geral para o fator "F" que facilita o cálculo das prestações periódicas de um financiamento.

Os exemplos hipotéticos comprovam a simplicidade de sua aplicação nas mais diferentes formas de vendas a prazo praticadas no mercado.

Considerando-se que existem as mais diferentes formas de financiamento o resultado aqui obtido será útil a um grande número de profissionais e empresas que prestam seviços ou vendem mercadorias a prazo

\section{BIBLIOGRAFIA:}

01. GIL, Antonio Carlos. Projetos de Pesquisa, Atlas S/A, São Paulo, 1993. 159p

02. VERAS, Lilia Ladeira. Matemática Financeira, Atlas S/A, São Paulo, 1989. 268p.

03. JUER, Milton. Matemática financeira: Aplicações no mercado de títulos, IBMEC, Rio de Janeiro, 1985. 483p 
